\title{
Synthesis and antibacterial activity of new 4"-O-carbamates of 11,12-cyclic carbonate erythromycin A 6,9-imino ether
}

\author{
Ling Zhang, Bo Jiao, Xiangrui Yang, Lin Liu and Shutao Ma
}

The Journal of Antibiotics (2012) 65, 115; doi:10.1038/ja.2011.129

Correction to: The Journal of Antibiotics (2011) 64, 243-247; doi:10.1038/ja.2010.166

The authors of the above article have rewritten the calculated and measured values of HR-MS, which were inaccurate in the publication of this paper. The correct calculated and measured values of compounds $\mathbf{5 a} \mathbf{a}-\mathbf{i}$ are presented below.

5a:

HR-MS (ESI) $m / z$ calculated for $\mathrm{C}_{47} \mathrm{H}_{74} \mathrm{~N}_{3} \mathrm{O}_{15}[\mathrm{M}+\mathrm{H}]^{+}$: 920.5120, found 920.5114 .

5b:

HR-MS (ESI) $m / z$ calculated for $\mathrm{C}_{46} \mathrm{H}_{71} \mathrm{FN}_{3} \mathrm{O}_{14}[\mathrm{M}+\mathrm{H}]^{+}$: 908.4920, found 908.4902 .

5c:

HR-MS (ESI) $m / z$ calculated for $\mathrm{C}_{46} \mathrm{H}_{71} \mathrm{ClN}_{3} \mathrm{O}_{14} \quad[\mathrm{M}+\mathrm{H}]^{+}$: 924.4625, found 924.4603 .

5d:

HR-MS (ESI) $m / z$ calculated for $\mathrm{C}_{46} \mathrm{H}_{71} \mathrm{ClN}_{3} \mathrm{O}_{14} \quad[\mathrm{M}+\mathrm{H}]^{+}$: 924.4625, found 924.4628 . 5e:

HR-MS (ESI) $m / z$ calculated for $\mathrm{C}_{46} \mathrm{H}_{72} \mathrm{~N}_{3} \mathrm{O}_{14}[\mathrm{M}+\mathrm{H}]^{+}$: 890.5014, found 890.5007 .

5f:

HR-MS (ESI) $m / z$ calculated for $\mathrm{C}_{47} \mathrm{H}_{74} \mathrm{~N}_{3} \mathrm{O}_{14}[\mathrm{M}+\mathrm{H}]^{+}$: 904.5171, found 904.5149 .

5g:

HR-MS (ESI) $m / z$ calculated for $\mathrm{C}_{42} \mathrm{H}_{72} \mathrm{~N}_{3} \mathrm{O}_{14}[\mathrm{M}+\mathrm{H}]^{+}:$: 842.5014, found 842.5042 .

5h:

HR-MS (ESI) $m / z$ calculated for $\mathrm{C}_{43} \mathrm{H}_{74} \mathrm{~N}_{3} \mathrm{O}_{14}[\mathrm{M}+\mathrm{H}]^{+}:$: 856.5171, found 856.5195 .

5i:

HR-MS (ESI) $m / z$ calculated for $\mathrm{C}_{42} \mathrm{H}_{70} \mathrm{~N}_{3} \mathrm{O}_{14}[\mathrm{M}+\mathrm{H}]^{+}:$: 840.4858, found 840.4895 . 